\title{
Experimental Modal Analysis of A Car Semi-Axle
}

\author{
Dejian-Meng ${ }^{1, *}$ \\ 1.Tianjing University of Technology and Education, \\ Tianjin 300222,China;
}

\author{
Hailong-Deng ${ }^{2}$ \\ 2.Tangshan yanshan iron and steel co. LTD, \\ Tangshan 064400,China
}

\begin{abstract}
Semi-axle is an important part of automobile. In the free state, the test instrument OROS was used to test the dynamic performance of a certain automobile semi-axle. The experimental modal parameters were obtained by using the multi-reference point least square complex frequency domain method, and it was verified by the modal confidence (MAC). The method and results of this study have important reference for studying dynamic performance of automotive semi-axle.
\end{abstract}

Keywords: Semi-axis; Test instruments; Modal parameters

\section{INTRODUCTION}

With the rapid development of economy and industry, the vehicle is moving towards high speed and comfortable direction, which directly leads to the increasingly violent mechanical vibration[1]. And now car enterprises frequently upgrade and transform the automobile, making the working conditions of the semi-axle more complex. The semi-axle is an important part of vehicle transmission system[2]. Whether the design of semi-axle is reliable directly affects the power transmission. Therefore, the dynamic performance test is carried out to better understand its natural frequency, mode and damping, providing reference for avoiding resonance to improve service life[3].

\section{THE THEORETICAL BASIS OF MODAL ANALYSIS}

$$
M \ddot{x}+C \dot{x}+K x=f
$$

$M$ is the mass matrix, $C$ is the damping matrix, $K$ is the stiffness matrix, $f$ is the load vector, $f$ and $x$ is a function of time.

$f=0$,applying the Laplace transform to the left and right sides of equation (1), and get it:

$$
\left(s^{2} M+s C+K\right) X(s)=F(\mathrm{~s})
$$

$X(s)$ is the displacement response, $F(s)$ is the Laplace transform of excitation force.

$$
Z(s)=s^{2}+s C+K
$$

The formula (2) and (3) can be written:

$$
X(s)=Z(s)^{-1} F(s)=H(s) F(s)
$$

(4) $H(S)$ is the transfer

function matrix

The elements of $l$ rows and $p$ columns in the transfer function can be expressed as:

$$
H_{l, p}(w)=\sum \frac{\phi_{l r} \phi_{p r}}{m_{r}\left[\left(w^{2}{ }_{r}-w^{2}\right)+j 2 \xi_{r} w_{r} w\right]}
$$

$w_{r}^{2}=\frac{k_{r}}{m_{r}}, \xi_{r}=\frac{c_{r}}{2 m_{r} w_{r}}, m r$ is the modal mass of the $r$ order, $w r$ is the modal frequency of the $r$ order, $r$ is the damping ratio of the $r$ mode, $r$ is the modal mode of the $r$ order[4]. 


\section{THE SEMI-AXIAL MODAL TEST}

OROS V3 dynamic signal analyzer, NVGATE and Modal II analysis software were used in this experiment.

\subsection{Test model}

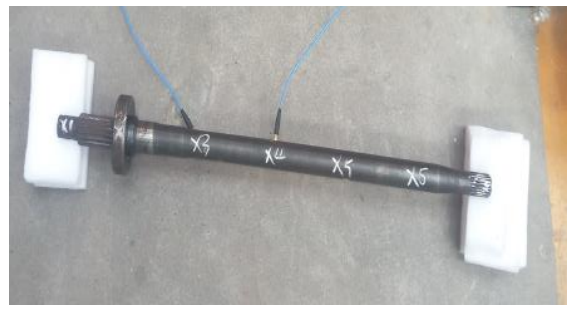

Figure.1 Half-axis real figure
Figure.2 Semi-axle test model

Figure 1 is the semi-axial physical figure of the experiment, and figure 2 is the semi-axial experimental model made by using modal analysis software.

\section{THE TEST RESULTS}

\subsection{Parameter identification}

The measured data were comprehensively analyzed by using modal analysis software, and the frequency response function in figure 3 and the frequency response function steady-state diagram in figure 4 were shown.

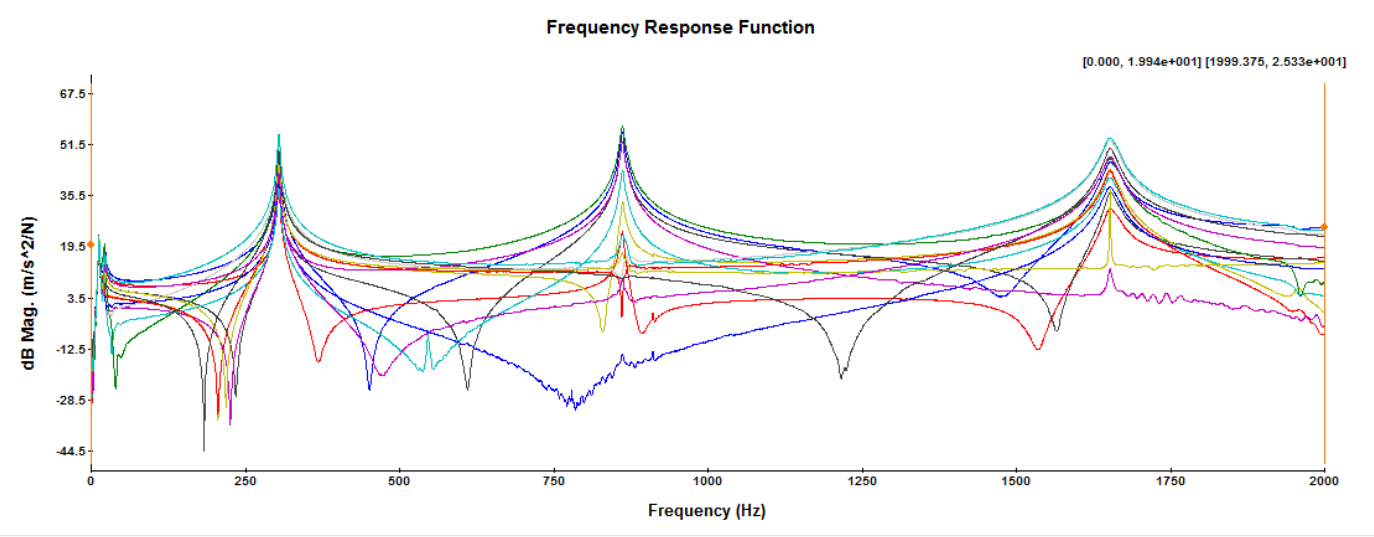

Figure.3 Frequency response function 


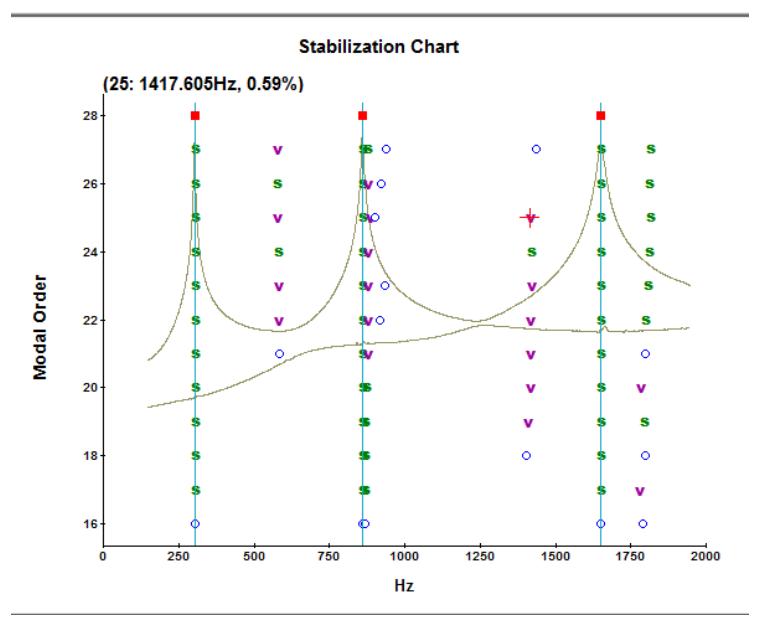

Figure.4 Steady-state diagram of the frequency response function

According to the frequency response function and the steady-state graph in figure 3 and figure 4, corresponding modal recognition can be performed to obtain the natural frequency of each order within $2000 \mathrm{~Hz}$ in table 1 and mode of vibration in figure 5 .

Table 1

\begin{tabular}{|c|c|c|}
\hline Modal & Frequency & Damping ratio \\
\hline 1 & 304.18 & 0.41 \\
\hline 2 & 861.35 & 0.35 \\
\hline 3 & 1652.01 & 0.60 \\
\hline
\end{tabular}
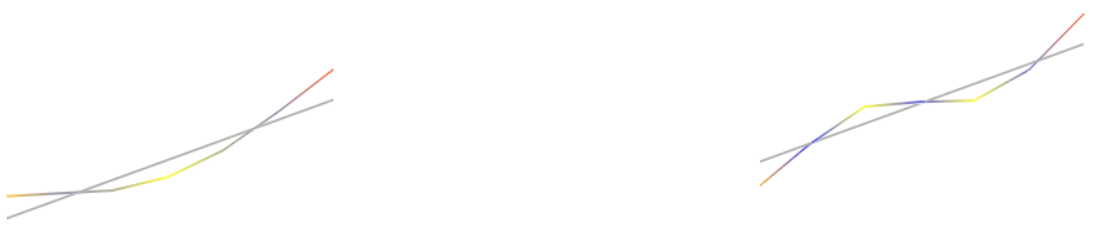

First order bending

Second order bending

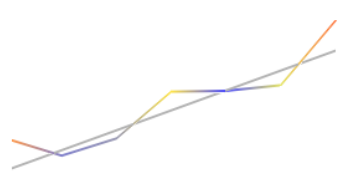

Third order bending

Figure .5 Variation diagram of mode of vibration 
It can be seen from figure 5 that the first mode is the bending vibration of two fixed points, while the first mode is the bending vibration of three fixed points, and the third mode is the bending vibration of four fixed points[5].

\subsection{Parameter validatio}

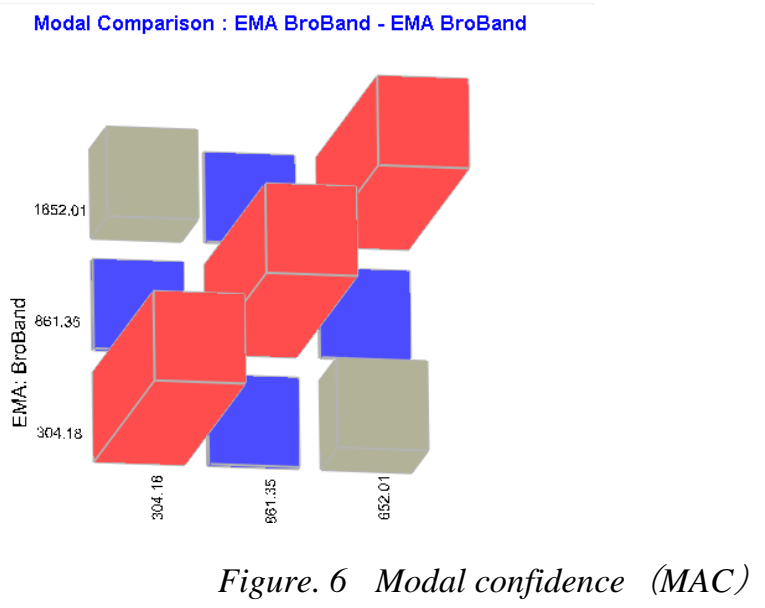

It can be seen from figure 6 that there is no similarity between all modes in the recognized mode 3 , indicating that the test point and sensor bonding are reasonable and there is no close mode or false mode.

\section{CONCLUSION}

By testing the vibration of the semi-axle in free state, the effects of dynamic characteristics such as vibration mode, damping and natural frequency on the semi-axle performance and vibration response characteristics can be obtained. The following conclusions can be obtained by analyzing the modal shapes and corresponding frequencies of each mode and the experimental data under MAC verification:

(1)It is feasible to use foam (sponge) to support the semi-axle in order to make the semi-axle free, which provides a basis for reasonably supporting experimental materials and obtaining better modal parameters.

(2)It can be seen from the experiment that the corresponding frequency of mode 2, 3, 4 and 5 of the semi-axle is above $1000 \mathrm{~Hz}$, which is much larger than the actual vibration frequency of the semi-axle, while the natural frequency of the object is inversely proportional to the toughness, indicating that the semi-axle has a small toughness, which provides theoretical guidance for its design modification and structural optimization.

\section{REFERENCES}

[1] Ding weiqi et al(2011). Light weight design of a heavy vehicle axle housing based on topology optimization. Automotive practical technology.

[2] Wu yundong, li renxian, ding weiping, kang qiang, Yang mingliang, xiang wei(2016). New micro-passenger rumble management based on semi-axle torsional stiffness adjustment . Noise and vibration control, vol.36, no.1,p.70-74+78. Communications, vol.78, no.1, p.231-246.

[3] Kang guidong, huang yingyun, liu peng(2011). Experimental modal study of crankshaft of TBD234V6 diesel engine. Small internal combustion engine and motorcycle,vol.40, no.4,p.31-34.

[4] Li deshui, yan bing, hua chunrong, dong dawei, wang jingxin(2010). Experimental study on crankshaft mode of small diesel engine. Automotive engine, no.2,p.89-92.

[5] Liao renwei(2014). Design and manufacturing process and experimental study of flexible flywheel of automobile engine. Chongqing university. 